\title{
Restoration of Hypothenar Muscle Function in Ulnar Nerve Paralysis*
}

\author{
D. A. RANNEY $\dagger$ \\ Schieffelin Leprosy Research Sanatorium, \\ Karigiri, S. India
}

\begin{abstract}
Insufficient attention has been given to the loss of hypothenar function in ulnar nerve paralysis. While restoration of a normal sequence of grasp and a strong pinch are of paramount importance in all patients, it is also important in certain cases to give a cosmetically acceptable shape to the hand for purposes of communication-a long-neglected function of the hand. Reference is made to a new technique using the extensor digiti minimi which, by reconstructing the transverse metacarpal arch not only has cosmetic value, but also produces a useful 5 -finger pinch. Being an extensor, it cannot improve strength of grip-a third function of the hypothenar muscles-but increased grip strength per se is not necessarily desirable in anaesthetic hands. Finally, the complex interrelationship between the mobility of the transverse metacarpal arch and the effect of lumbrical replacement procedures is briefly discussed.
\end{abstract}

Grasp and pinch are the two most useful functions of the hand. The hand is also a sense organ. But an of forgotten use of the hand is in communication (Table 1).

TABLE 1

Four functions of the hand

\begin{aligned} & \hline 1. Grasp \\ & 2. Pinch-digits 2, 3, and 5 \\ & 3. As a sense organ \\ & 4. Communication of ideas \\ & \hline\end{aligned}

For this purpose the hand must have graceful mobility and a pleasing shape. A badly shaped hand has limited function as a means of communication. This fact is particularly important in countries where there is still a serious degree of social rejection of leprosy sufferers, for the shape of the hand may communicate the fact that the speaker has leprosy. Therefore, it is essential that in the practice of leprosy reconstructive surgery we should direct our efforts toward the elimination of such cosmetic defects, while at the same time restoring a normal sequence of grasp and a strong pinch.

We cannot speak of form as distinct from function, for in order to function well as a means of communication the hand must have a cosmetically acceptable form. The value of this particular function will vary according to the rôle of the

* Paper read at the Tenth International Leprosy Congress, Bergen, Norway, 1973.

† From September 1973, Visiting Assistant Professor of Anatomy, Queens University, Kingston, Ontario, Canada. 
patient in society and the degree of acceptance or rejection he is likely to experience. It will therefore be of so little value to a farmer in a society that accepts leprosy patients as not to matter; but to one who is daily exhibiting his hands to a more hostile society, e.g. a bank clerk in an urban area, the shape of his hands will determine whether or not he is accepted for employment.

Standard operations are available for lumbrical replacement and for restoration of thumb action. In those operations designed to improve grasp and pinch the importance of cosmesis is generally recognized. Efforts are also being made to restore sensation where possible-although much more remains to be done in this field. But in all our operations we neglect the important functions of the hypothenar muscles (Table 2). The restoration of hypothenar function is important in attempting to restore the function of grasp and pinch as well as to improve appearance. Perhaps a too brief account of the reason for this is given abóve, but a more adequate description will be published shortly (Ranney, 1973b).

TABLE. 2

Four functions of hypothenar muscles

\begin{tabular}{ll} 
1. & Cosmesis-Shape of the hand \\
2. & Opposition-Little finger to thumb \\
3. & Grasp-Increased pressure \\
4. & Grasp-Augmented lumbrical action \\
\hline
\end{tabular}

\section{Function of the Hypothenars}

\section{COSMESIS}

One of the cosmetic defects in ulnar paralysis is flattening of the transverse metacarpal arch due to paralysis of the hypothenar muscles. This can be usually aggravated by surgical correction of clawing of the fingers. The reason for this is given below (Fig. 1).

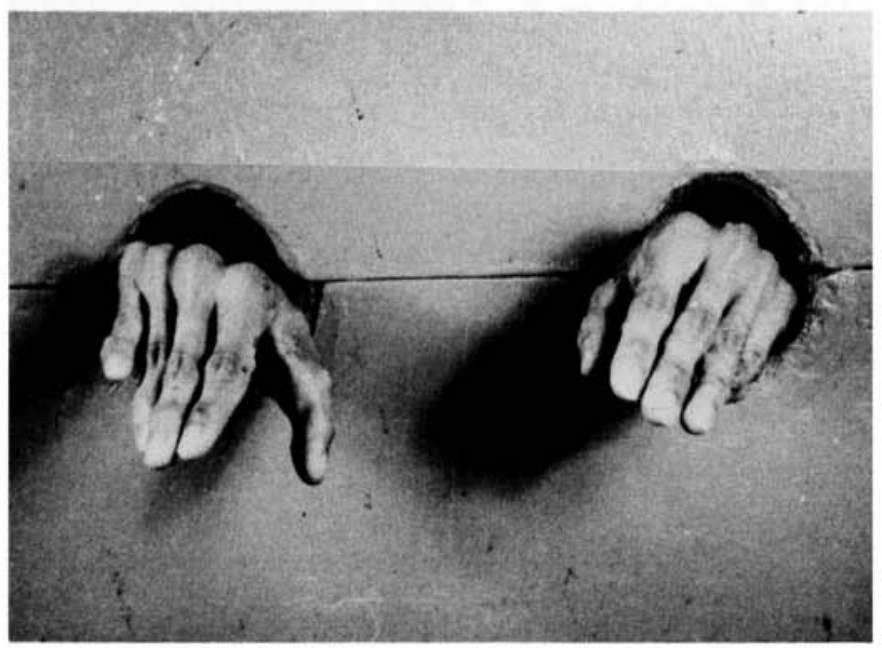

Fig. 1. A normal left hand and a right hand with arch reversal following a satisfactory extensor-flexor many-tailed operation. 
Normal appearance can be restored by a new operation to reconstruct the transverse metacarpal arch (Fig. 2) (Ramney, 1973a).

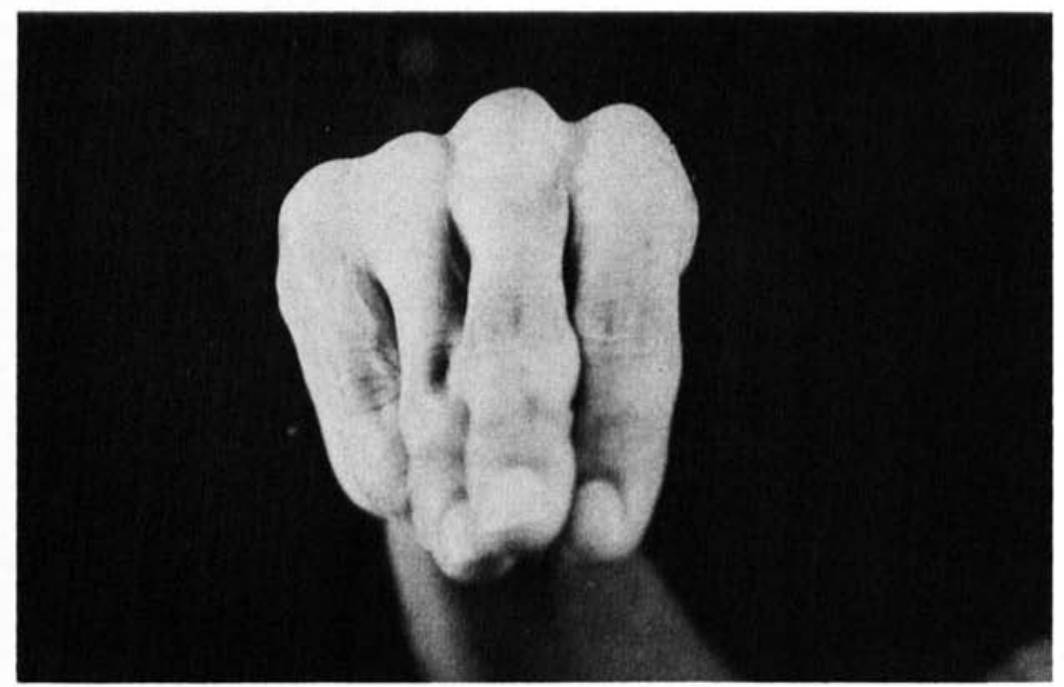

Fig. 2. Appearance of the arch after tendon transfer to restore hypothenar function-normal appearance restored.

\section{PINCH}

Cosmesis is not the only reason for wanting to restore the function of the hypothenar muscles. Any operation which increases the curvature of the transverse metacarpal arch simulates the action of opponens digiti minimi muscle by bringing the little finger into a functional relationship with the thumb (Fig. 3 ).

As Antia pointed out at the International Leprosy Colloquium held in Borstel (Antia, 1971) the possession of a 5-finger pinch is most important. Not only is it useful in Eastern countries while eating rice, but is also helpful in any society in picking up objects which approximate to the size of the palm.

\section{GRIP PRESSURE}

A third function of the hypothenar muscles is to improve grip pressure by protraction of the fifth metacarpal. Increase in grip pressure has not so far been achieved. Increase in pressure in the anaesthetic hand is not necessarily a good thing, but a more equitable distribution of pressure might be At present we are studying patterns of grip pressure, but it is too early to comment on this as yet.

\section{AUGMENTATION OF LUMBRICAL ACTION}

Fourthly, the hypothenar muscles augment the lumbrical action of the ring and little fingers. It is noteworthy that in cases of recurrent clawing, of which there are many possible causes, recurrence is most of ten seen in these two fingers that have mobile CMC joints. In any multiple-joint system it is necessary to have each mobile joint under control to prevent development of a zig-zag deformity (Landsmeer, 1958; Stack and Vaughan-Jackson, 1971). For instance, in lumbrical 


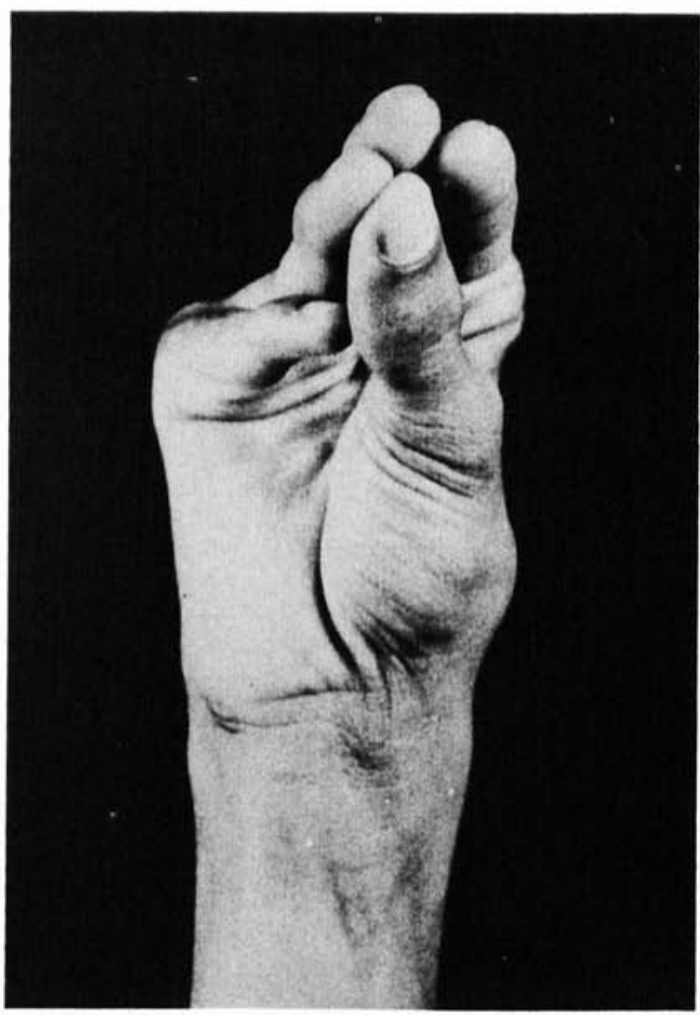

Fig. 3. The achievement of the 5-finger pinch after operation to restore the transverse metacarpal arch.

paralysis the metacarpo-phalangeal joint goes into hyperextension since there is no independent flexor of this joint (Fig. 4). The secondary result of this is flexion of the interphalangeal joints, and clawing results. By restricting metacarpophalangeal hyperextension, either with a tendon transfer (F3) or by passive means, the pull of the finger extensor is transferred to the interphalangeal joints and the zig-zag phenomenon is eliminated. However, this extensor force may be dissipated in extending the carpo-metacarpal joints if these joints are mobile.

Of course, we all know that, with "sufficient" tension on the lumbrical grafts to the little and ring fingers, recurrent clawing can be prevented, even in hands with a very mobile arch. But in so doing the arch is flattened or even reversed. This is a passive means of controlling the arch-permanently reversing it and keeping it that way. I believe there is a way which in some cases (not all) may be better and that is to add Landsmeer's third force, a tendon transfer to control mobility of the arch.

Figure 5 illustrates the fact that even passive restriction of metacarpophalangeal joint extension can cause arch reversal. This patient, a boy, had a Zancolli capsulorrhaphy of the lit tle finger. He had had clawing of the other 3 fingers when I saw him for the first time. Note that in the open hand position he has a considerable degree of limitation of extension. 


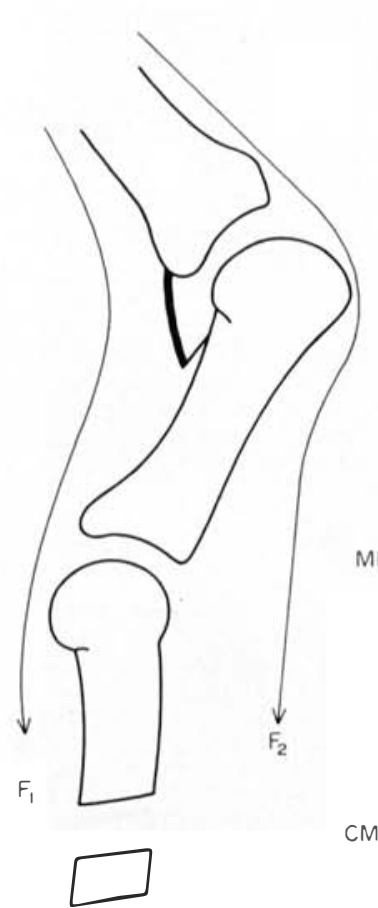

Zig-zag

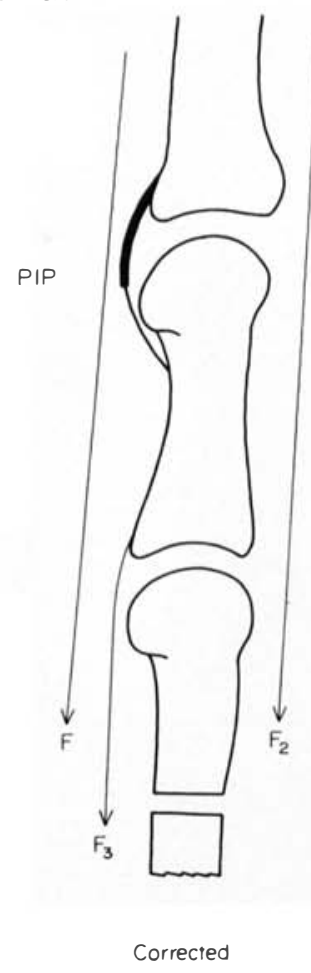

Fig. 4. When a third force is added the zig-zag deformity, in this case clawing of the finger, is corrected.

Note that he has also developed a severe arch reversal (Fig. 6). This is due to the fact that the extensor digiti minimi is so severely restricted in its ability to extend the metacarpo-phalangeal joint. The unused force is transferred to the fifth carpo-metacarpal joint, extending it and reversing the arch. Srinivasan reports a similar case.

It was this case that led the present author to consider using the extensor digiti minimi as a corrective force, while at the same time removing its deforming action on the transverse metacarpal arch. A detailed description of the technique is reported elsewhere (Ranney, 1973a) and it is important to follow these details in the performance of this operation.

\section{INTERRELATIONSHIP BETWEEN ARCH AND LUMBRICAL REPLACEMENT}

Operations to reconstruct the transverse metacarpal arch have yet to be tried on a large scale prior to lumbrical replacement. Theoretically, at least, it should help to prevent recurrent clawing (Ranney, 1973b). In the surgically corrected ulnar claw hand, the extensor expansion is drawn proximally by the combined action of the finger extensor tendons and the lumbrical replacement grafts. This effectively straightens the interphalangeal joints and prevents hyperextension of the metacarpo-phaleangeal joints in the index and long finger. But the motor to these fingers is shared in common with the ring and little fingers. Since the 


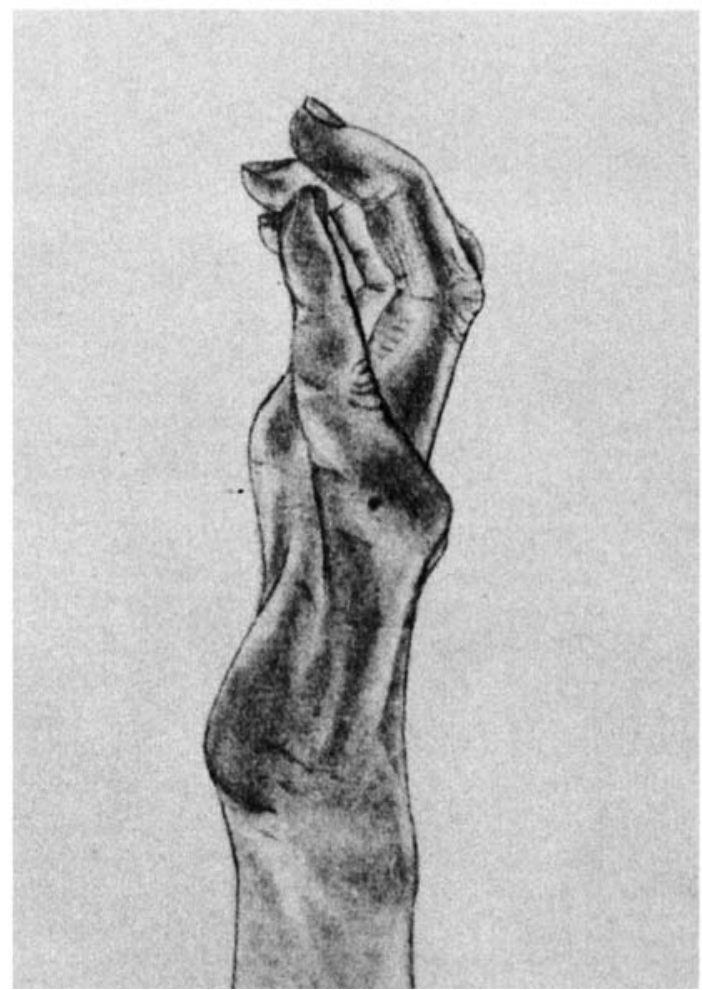

Fig. 5. Open hand position showing a considerable degree of extension limitation following Zancolli Capsulorrhaphy with the metacarpo-phalangeal joint.

metacarpals of the ring and little finger are mobile they can move backward into extension at the carpo-metacarpal joints. The result is that instead of pulling the sleeve-like extensor apparatus (Littler, 1967) proximally on them, the medial 2 fingers and their metacarpals are pulled backwards without sufficient extension of the interphalangeal joints taking place. This can occur after the extensor-flexor many-tailed operation as well as after the extensor-extensor procedure, although the reversal is greater in the latter situation because a second factor is operating (Ranney, 1973b).

There are two possibilities for remedying this situation, as Landsmeer pointed out many years ago-either supply a third force, or restrict the mobility of the extra joint by passive means (Landsmeer, 1958). The fifth metacarpal can be pulled forward with an active transfer, dragging the fourth in its wake. For this purpose I prefer the extensor-digiti-minimi transfer. The alternative is to put sufficient tension on the medial two slips so that the fourth and fifth carpo-metacarpal joints are permanently extended and the arch permanently reversed.

\section{Conclusion}

It is nonsense to think that, in a completely denervated hand, we can with 2 tendon transfers completely replace all the more useful functions of 19 intrinsic 


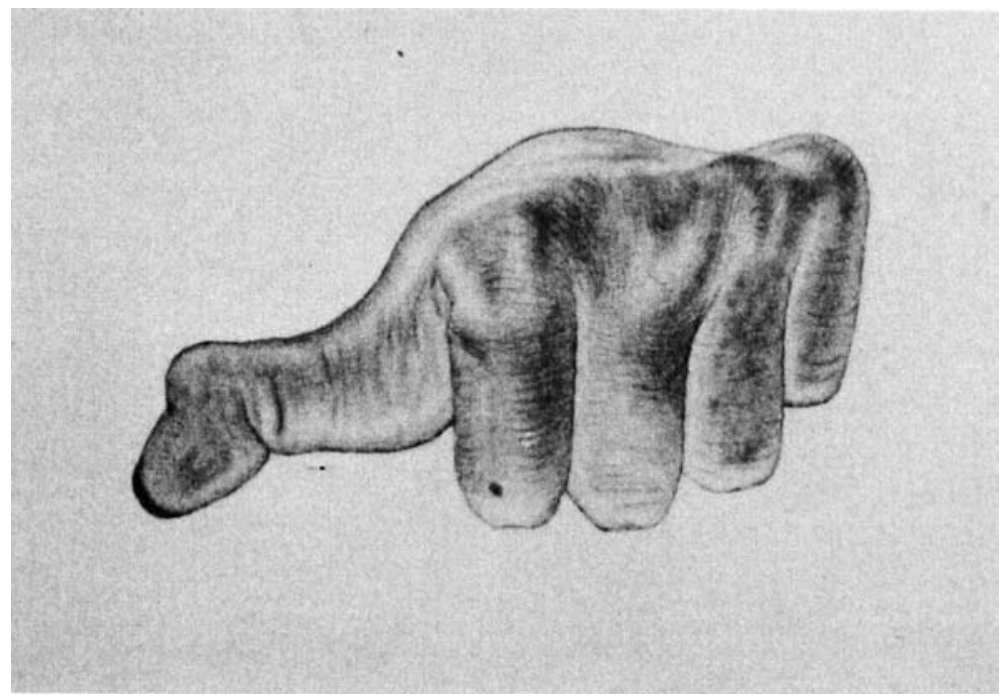

Fig. 6. View of the arch after Zancolli capsulorrhaphy; same patient as in Fig. 5 .

muscles. Restoration of thumb opposition and lumbrical action in the fingers is of paramount importance. But, in addition to this, at least three of the functions of the hypothenar muscles can and sometimes should be restored by an operation to control transverse metacarpal arch mobility. Anterior transposition of the extensor digiti minimi can do this. The author has not been entirely satisfied with this procedure in all cases. Perhaps better operations can be devised. The improved appearance and achievement of a 5-finger pinch are useful in those whose occupational status require it. However, the operation is not needed in all cases, as the majority of the patients are rural labourers. But if two of these functions of the hypothenars, namely cosmesis and a 5-finger pinch. are not required, it should be realized that some type of stabilization of the mobile metacarpals must be achieved in order to prevent recurrent clawing of the little and ring fingers. Such stabilization can be achieved by applying more tension on the two medial lumbrical grafts and accepting the inevitable flattening, or even frank reversal, of the arch that will result.

\section{References}

Antia, N. H. (1971). Review of surgery in leprosy. Int. J. Lepr. 39.616-617.

Landsmeer, J. M. F. (1958). A report on the co-ordination of the interphalangeal joints of the human finger and the disturbances. Acta Morph. Neerl. Scand. 2, 59-84.

Littler, W. (1967). The finger extensor mechanism. The Surgical Clinics of North A merica 47, 147-153.

Ranney, D. A. (1973a). Reconstruction of the transverse metacarpal arch in ulnar palsy by transfer of the Extensor Digiti Minimi.

Ranney, R. A. (1973b). The mechanism of arch reversal in the surgically corrected claw hand. In preparation.

Stack, H. G. and Vaughan-Jackson, O. S. (1971). The zig-zag deformity in the rheumatoid hand. The Hand 3, 62-67.

Srinivasan, H. Personal communication. 